\title{
A HOMOGENIZATION TECHNIQUE FOR THE BOLTZMANN EQUATION FOR LOW PRESSURE CHEMICAL VAPOR DEPOSITION*
}

\author{
MATTHIAS K. GOBBERT ${ }^{\dagger}$ AND CHRISTIAN RINGHOFER ${ }^{\ddagger}$
}

\begin{abstract}
We present a homogenization technique for rarefied gas flow over a microstructured surface consisting of patterns of periodic features. The length scale of the model domain is comparable to the mean free path of the molecules, while the scale of the surface patterns is much smaller. The flow is modeled by a system of linear Boltzmann equations with a diffusive boundary condition at the patterned surface. The resulting homogenized boundary condition holds at a virtual flat surface and incorporates the microscopic geometry information about the surface structure on the macroscopic level. Numerical results validate the approach. The setup models low pressure chemical vapor deposition processes in the manufacturing of integrated circuits.
\end{abstract}

Key words. Boltzmann equation, rarefied gas dynamics, boundary homogenization, microstructured surface, chemical vapor deposition

AMS subject classifications. 65M06, 76P05, 35B27, 76M45, 80A30

DOI. $10.1137 /$ S0036139902393476

1. Introduction. Low pressure chemical vapor deposition is used in the manufacturing of integrated circuits to deposit a thin layer of material onto the surface of a silicon wafer. The deposition surface necessarily involves a microstructure given by the electrical components of the future microchip. Classical models for this process include reactor scale models [17] with a typical length scale of over $10 \mathrm{~cm}$, which model the gas flow throughout the chemical reactor, and feature scale models [3] with a typical length scale of under $1 \mu \mathrm{m}$, which focus on the evolution of the film profile inside an individual feature.

In more detail, the process works as follows. Molecules of the species to be deposited are carried inside the chemical reactor by an inert carrier gas to a microstructured surface, where they are partially absorbed and partially reflected at a certain rate. The length scale of the surface structure is several orders of magnitude smaller than that of the reactor and therefore cannot be reasonably resolved on the reactor scale. On the other hand, this structure will influence the gas flow through the boundary conditions; i.e., adsorption on the microstructured surface will result in a different behavior of the gas flow than adsorption on a flat surface, even on the macroscopic reactor scale. We therefore have to solve a homogenization problem at the boundary by deriving a boundary condition for the flow problem which incorporates the microscopic geometric information about the surface structure into the macroscopic flow picture.

* Received by the editors October 11, 2002; accepted for publication (in revised form) April 15, 2003; published electronically November 19, 2003. This research was supported in part by the Institute for Mathematics and its Applications, with funds provided by the National Science Foundation. This research was also supported by the Wittgenstein Award 2000 of Peter Markowich, financed by the Austrian Research Fund.

http://www.siam.org/journals/siap/64-1/39347.html

${ }^{\dagger}$ Department of Mathematics and Statistics, University of Maryland, Baltimore County, 1000 Hilltop Circle, Baltimore, MD 21250 (gobbert@math.umbc.edu). The research of this author was supported in part by the National Science Foundation under grant DMS-9805547.

${ }^{\ddagger}$ Department of Mathematics and Statistics, Arizona State University, Tempe, AZ 85287-1804 (ringhofer@asu.edu). The research of this author was supported in part by the National Science Foundation under grant DMS-9706792. 
Based on analytic work in $[2,5,6,8,11]$, the authors and coworkers have previously introduced $[7,12]$ a mesoscopic scale model on a length scale intermediate to those of the classical reactor scale and feature scale models, which was designed to provide information on the effects of feature clustering on a length scale of about $1.0 \mathrm{~cm}$ at comparably high pressures of at least 1 torr (where 760 torr $=1 \mathrm{~atm}$ ). In that regime, the mean free path of the gas molecules was well inside the domain size, and the gas flow could be modeled as diffusion-dominated $[8,9,11,12,18]$. While $[8,11]$ derive the homogenized model formally for the concrete problem of interest, $[2,5,6]$ handle the analysis rigorously for a more general class of models.

However, as individual feature sizes decrease below $1 \mu \mathrm{m}$, the length scale of interest for clustering effects also decreases. Therefore, this work considers a mesoscopic scale model with a domain with typical length scale on the order of $0.01 \mathrm{~cm}$. Using this together with typical values for the total pressure of 1 torr or less, the mean free path of the gas molecules is of length comparable to the typical length scale. The Knudsen number Kn, which is defined as the ratio of the mean free path and the typical length scale, is on the order of unity, and the process lies in the transition regime for gaseous flow modeling [15].

The proper mathematical model for a gas flow in the transition regime is given by the Boltzmann equation of gas dynamics for the (scaled) probability density $f(x, v, t)$ that there is a molecule in the region $\left[x_{1}, x_{1}+d x_{1}\right] \times\left[x_{2}, x_{2}+d x_{2}\right] \times\left[x_{3}, x_{3}+d x_{3}\right]$ with velocity in $\left[v_{1}, v_{1}+d v_{1}\right] \times\left[v_{2}, v_{2}+d v_{2}\right] \times\left[v_{3}, v_{3}+d v_{3}\right]$ during time $[t, t+d t]$,

$$
\frac{\partial f}{\partial t}+v \cdot \nabla f=Q(f, f)
$$

with the collision operator

$$
Q(f, f)(x, v, t)=\iiint\left[f\left(x, v^{\prime}, t\right) f\left(x, v_{*}^{\prime}, t\right)-f(x, v, t) f\left(x, v_{*}, t\right)\right] B(\vartheta,|V|) d \vartheta d \varepsilon d v_{*},
$$

where $v^{\prime}=v-n(n \cdot V)$ and $v_{*}^{\prime}=v_{*}+n(n \cdot V)$ denote the precollision velocities, with $V=v-v_{*}$ and $n=(\sin \vartheta \cos \varepsilon, \sin \vartheta \sin \varepsilon, \cos \vartheta)^{T}($ see $[4,15])$.

A complete model for chemical vapor deposition will consist of one Boltzmann equation for each gaseous species $f_{i}(x, v, t), i=0,1, \ldots, n_{s}$, to form the system

$$
\frac{\partial f_{i}}{\partial t}+v \cdot \nabla_{x} f_{i}=\sum_{j=0}^{n_{s}} Q_{i j}\left(f_{i}, f_{j}\right), \quad i=0,1, \ldots, n_{s}
$$

where the collision operators $Q_{i j}\left(f_{i}, f_{j}\right)$ model the collisions between molecules of species $i$ and species $j$ for $0 \leq i, j \leq n_{s}$. This model includes the inert background gas $f_{0}(x, v, t)$ of the manufacturing process, which is a rarefied gas itself but still much denser than the reacting species. Under these assumptions, the collisions of a reacting species with the background gas will be much more frequent than collisions among the reacting species, and it is legitimate to neglect all collisions except the ones with the background species $j=0$ on the right-hand side of (1.3); this also decouples the equation for the background species $f_{0}$ from the other equations, which can hence be solved for independently from the other solutions $f_{i}, i=1, \ldots, n_{s}$.

In the classical derivation [4, Chapter IV], it is additionally assumed that the background gas is in equilibrium and spatially homogeneously distributed. Then its 
distribution function $f_{0}(x, v, t)$ is given by a Maxwellian distribution of the form

$$
f_{0}(x, v, t)=M(v):=\frac{1}{(2 \pi)^{d / 2}} \exp \left(-\frac{|v|^{2}}{2}\right),
$$

where $d \in\{1,2,3\}$ denotes the dimension of the velocity space under consideration. Then, the time-evolution of the probability distribution of a typical reacting species is given by the system of linear Boltzmann equations

$$
\frac{\partial f_{i}}{\partial t}+v \cdot \nabla f_{i}=Q_{i}\left(f_{i}\right), \quad i=1, \ldots, n_{s}
$$

with the linear collision operators

$$
Q_{i}\left(f_{i}\right)(x, v, t)=\int S_{i}\left(v, v^{\prime}\right)\left[\frac{f_{i}\left(x, v^{\prime}, t\right)}{M\left(v^{\prime}\right)}-\frac{f_{i}(x, v, t)}{M(v)}\right] d v^{\prime}
$$

see $\left[4\right.$, Chapter IV] for a detailed derivation. Here, $S_{i}\left(v, v^{\prime}\right)=S_{i}\left(v^{\prime}, v\right)$ denotes the scattering cross section, which describes the probability that a molecule with velocity $v^{\prime}$ before a collision scatters to a velocity $v$ after the collision. A generalization to spatially varying background gases is possible. We will assume that the reacting species are introduced into the reactor chamber starting at the beginning of the processing step, i.e., that $f_{i}=0, i=1, \ldots, n_{s}$, at $t=0$.

For the analysis, we therefore consider the representative equation for $f(x, y, v, t)$ :

$$
\frac{\partial f}{\partial t}+v_{1} \frac{\partial f}{\partial x_{1}}+v_{2} \frac{\partial f}{\partial x_{2}}+v_{3} \frac{\partial f}{\partial y}=Q(f)
$$

Here, $x \equiv\left(x_{1}, x_{2}\right)^{T}$ counts across the reacting surface, and $y \equiv x_{3}$ points into the gaseous domain perpendicular to the surface. That is, molecules with velocities $v=$ $\left(v_{1}, v_{2}, v_{3}\right)^{T}$ travel towards the mean wafer surface when $v_{3}<0$. More precisely, the microstructured surface $\Gamma_{\varepsilon}$ is for this paper assumed to be given by a function

$$
y=\tilde{h}(x)=\varepsilon h\left(x, \frac{x}{\varepsilon}\right),
$$

with the small parameter $0<\varepsilon \ll 1$. This parameter represents the ratio of the typical feature mouth (or, more precisely, the so-called pitch, i.e., the distance from the center of one feature to the next one), e.g., $1 \mu \mathrm{m}$, to the typical length scale of the mesoscopic scale model, e.g., $100 \mu \mathrm{m}$.

In this dimensionless form, $h(x, \xi)$ is periodic in $\xi \equiv\left(\xi_{1}, \xi_{2}\right)^{T}$ with period 1 in each component; that is,

$$
h(x, \xi)=h\left(x, \xi+e_{1}\right)=h\left(x, \xi+e_{2}\right) \quad \text { for all }(x, \xi),
$$

where $e_{1}=(1,0)^{T}$ and $e_{2}=(0,1)^{T}$ denote the unit vectors in two dimensions. Physically, this reflects the fact that the microscopic features on a computer chip are not random but arranged in clusters of hundreds or thousands of (by design) identical features, periodic with the feature pitch as period. However, this periodic structure will be different in different regions of the chip, and therefore we allow the function $h$ to depend on the slow spatial variable $x$ as well. Realistic values for $\varepsilon$ include the range from $10^{-4}$ to $10^{-3}$.

The model is complemented by a boundary condition on the reacting surface for all molecules that flow into the gaseous domain, i.e., that satisfy $n \cdot v \leq 0$, where $n=$ 
$n(x, y)$ denotes the unit outward normal vector at position $(x, y)^{T} \in \Gamma_{\varepsilon}$. Specifically, we assume that the reinsertion occurs with Maxwellian distributed velocities and set

$f(x, y, v, t)=M(v) \int_{n \cdot w>0} a\left(x, \frac{x}{\varepsilon}, w\right) f(x, y, w, t) d w \quad$ for $n \cdot v \leq 0$ and $(x, y)^{T} \in \Gamma_{\varepsilon}$,

where $a\left(x, \frac{x}{\varepsilon}, w\right) \geq 0$ denotes a given function, and $n$ is the unit outward normal vector on the surface $\Gamma_{\varepsilon}$. This boundary condition reflects a pseudo-steady-state assumption, that is, the deposition of molecules on the surface progresses several orders of magnitude more slowly than the flow of the rarefied gas; therefore neither the functions $a\left(x, \frac{x}{\varepsilon}, w\right)$ nor the geometry of the surface $y=\tilde{h}(x)$ depends on the time $t$ of the gas flow under consideration. However, they are certainly allowed to depend on $x$; that is, different regions of the surface can see different deposition conditions; this is important in actual applications.

The model in its present form with a microstructured surface is not numerically tractable because of the high cost of resolving the domain close to the rough surface $\Gamma_{\varepsilon}$. The goal of this work is to obtain a model with a reduced boundary condition on a flat surface $\Gamma_{0}$ given by $y=0$ that gives equivalent results for $f(x, y, v, t)$ in the bulk of the gaseous domain away from the surface in an asymptotic sense using the expansion parameter $\varepsilon$.

To this end, we make the ansatz

$$
f(x, y, v, t)=\tilde{f}(x, y, v, t)+\hat{f}\left(\frac{x}{\varepsilon}, \frac{y}{\varepsilon}, \frac{t}{\varepsilon}, x, v, t\right)+o(1),
$$

where $\tilde{f}$ denotes the bulk variable, for which we wish to derive a numerically tractable model, and $\hat{f}$ is the small-scale correction, which is assumed to be periodic in $\xi=\frac{x}{\varepsilon}$ in the same way as the surface function $h(x, \xi)$. There is only one scale for the velocity $v$ of the molecules; hence there have to be pairs of corresponding length and time scales on both the long $(x$ and $t)$ and the short scales $\left(\xi=\frac{x}{\varepsilon}\right.$ and $\left.\tau=\frac{t}{\varepsilon}\right)$. Note that we have assumed the Knudsen number to be of order $O(1)$ in the bulk of the mesoscopic scale model, whose solution is $f(x, y, v, t)$. This means that on the $O(\varepsilon)$ spatial scale, the feature scale, collisions will be negligible, and we obtain free transport inside the features of the surface. Therefore, due to the hyperbolic nature of the Boltzmann equation, we can assume only that $\hat{f}$ decays weakly with $\varepsilon$ at any fixed distance from the surface; that is, we assume that small-scale fluctuations in the inner solution $\hat{f}$ average out to zero at any fixed finite distance above the surface as $\varepsilon \rightarrow 0$. That is, we require that

$$
\lim _{\varepsilon \rightarrow 0} \iint \hat{f}\left(\frac{x}{\varepsilon}, \frac{y}{\varepsilon}, \frac{t}{\varepsilon}, x, v, t\right) \psi(x, t) d x d t=0
$$

for all test functions $\psi(x, t)$.

Based on these assumptions, we will derive the appropriate reduced boundary condition for the bulk term $\tilde{f}(x, y, v, t)$ on the flat surface $y=0$

$$
\tilde{f}(x, y, v, t)=M(v) \int \tilde{a}(x, v, w) \tilde{f}(x, y, w, t) d w \quad \text { for } v_{3}>0 \text { and } y=0,
$$

where the integral kernel $\tilde{a}(x, v, w)$ incorporates the information about the microscopic surface geometry into the flow equations. This problem is tractable numerically, since it is posed on a domain with a flat reacting surface, and the values of $\tilde{a}(x, v, w)$ can be 
precomputed for all times, since $\tilde{a}$ does not depend on the macroscopic time $t$. This effective boundary condition still reflects the assumption of a pseudo-steady state in the original model, because $\tilde{a}$ does not depend on the time $t$ that is the relevant time scale for the gas flow on the mesoscopic scale.

We remark that in the previous work $[2,5,6,8,11]$, where the flow was assumed to be Maxwellian inside the features as well, the only information needed about the surface geometry was the ratio of surface areas between the microstructured surface and the flat surface. This ratio is now replaced by the integral kernel $\tilde{a}$, which contains much more information about the actual shape of the surface and is necessary for the non-Maxwellian picture. This approach has to be viewed as an alternative to the work presented in [1], which deals with specular reflections on a random surface, as opposed to random reflections on a deterministic surface. The result is, however, quite different for the obvious reason that we allow for absorption into the surface; i.e., in our resulting homogenized problem the total mass inside the gas phase domain will not be conserved.

Section 2 details the analytical derivation of the reduced boundary condition, where we will restrict ourselves to the two-dimensional case for notational simplicity. The generalization to three dimensions is straightforward. Section 3 provides a numerical demonstration of the result for a setup that closely resembles the structure of the application problem under consideration by using a periodic boundary geometry.

2. Analysis. This section considers the two-dimensional linear Boltzmann equation

$$
\frac{\partial f}{\partial t}+v_{1} \frac{\partial f}{\partial x}+v_{2} \frac{\partial f}{\partial y}=Q(f)
$$

with boundary condition (1.10) at the reacting wafer surface. Note that we have changed the notation slightly in going to a two-dimensional problem: The gas phase domain is now given by $y>\varepsilon h\left(x, \frac{x}{\varepsilon}\right)$, and molecules travel towards the surface for velocities $v=\left(v_{1}, v_{2}\right)^{T}$ with $v_{2}<0$. We introduce the surface density $\rho$ as

$$
\rho\left(\frac{x}{\varepsilon}, \frac{t}{\varepsilon}, x, t\right)=\int_{n \cdot w \geq 0} a\left(x, \frac{x}{\varepsilon}, w\right)\left(\tilde{f}(x, 0, w, t)+\hat{f}\left(\frac{x}{\varepsilon}, h\left(x, \frac{x}{\varepsilon}\right), \frac{t}{\varepsilon}, x, w, t\right)\right) d w+o(1)
$$

for the integral on the right-hand side of (1.10). Using the ansatz $f=\tilde{f}+\hat{f}+o(1)$, the boundary condition (1.10) then reads

$$
\tilde{f}(x, 0, v, t)+\hat{f}(\xi, h(x, \xi), \tau, x, v, t)=M(v) \rho(\xi, \tau, x, t)+o(1) \quad \text { for } \sigma \leq 0,
$$

using also the shorthand notation $\sigma(x, \xi, v)=n \cdot v=v_{1} \partial_{\xi} h(x, \xi)-v_{2}+\mathcal{O}(\varepsilon)$. Here we have already replaced the argument $y=\varepsilon h(x, \xi)$ by 0 in the evaluation of $\tilde{f}$ at the boundary. The homogenized boundary condition (1.13) on the limiting flat surface $y=0$ will be as derived follows.

Tracing back the characteristics, we will first solve the boundary layer problem for the inner solution $\hat{f}$ in terms of boundary data consisting of the outer solution $\tilde{f}$ and the boundary density $\rho$ defined in (2.2). This result is given in Theorem 2.2 in subsection 2.1. The surface density $\rho$ involves both the inner and the outer solutions and forms the connection between them. Its behavior in the limit of the fast time variables $\tau \rightarrow \infty$ is analyzed in Theorem 2.4. 
In subsection 2.2, using the weak decay condition (1.12), we derive the homogenized boundary condition for $\tilde{f}$ on the flat surface $y=0$ in terms of the surface density $\rho$ and finally in terms of $\tilde{a}$ as in (1.13). This quantity will contain only information about the microscopic and quasi-periodic geometry of the surface and can be solved beforehand for a given surface structure.

2.1. Solution to the inner equation. The asymptotic ansatz applied to (2.1) leads to the inner problem for the layer correction term $\hat{f}(\xi, \eta, \tau, x, v, t)$ as a function of the layer variables $\xi, \eta, \tau$ :

$$
\frac{\partial \hat{f}}{\partial \tau}+v_{1} \frac{\partial \hat{f}}{\partial \xi}+v_{2} \frac{\partial \hat{f}}{\partial \eta}=0
$$

for any fixed $x, v, t$. Using the method of characteristics with $\xi^{\prime}=v_{1}$ and $\eta^{\prime}=v_{2}$ yields

$$
\hat{f}(\xi, \eta, \tau, x, v, t)=\hat{f}\left(\xi-s v_{1}, \eta-s v_{2}, \tau-s, x, v, t\right)
$$

for all parameters $s$ sufficiently small. We can then follow the characteristics back to the boundary or back to the initial condition $\hat{f}=0$ at $\tau=0$ to obtain

$$
\begin{aligned}
\hat{f} & (\xi, \eta, \tau, x, v, t) \\
& =\hat{f}\left(\xi-v_{1} s, \eta-v_{2} s, \tau-s, x, v, t\right) \\
& = \begin{cases}\hat{f}\left(\xi-v_{1} \phi_{0}, \eta-v_{2} \phi_{0}, \tau-\phi_{0}, x, v, t\right) & \text { if } \phi_{0}<\tau, \\
0 & \text { if } \phi_{0} \geq \tau\end{cases} \\
& =H\left(\tau-\phi_{0}\right) \hat{f}\left(\xi-v_{1} \phi_{0}, \eta-v_{2} \phi_{0}, \tau-\phi_{0}, x, v, t\right),
\end{aligned}
$$

with

$$
\phi_{0}(\xi, \eta, x, v)=\left\{\begin{array}{l}
\min \left\{s>0: \eta-v_{2} s=h\left(x, \xi-v_{1} s\right)\right\} \\
\infty \text { if } \eta-v_{2} s \neq h\left(x, \xi-v_{1} s\right) \text { for all } s>0
\end{array}\right.
$$

and using the Heaviside function

$$
H(z)= \begin{cases}0 & \text { for } z<0 \\ 1 & \text { for } z \geq 0\end{cases}
$$

The function $\phi_{0}$ denotes the intersection time, i.e., the time it takes for a molecule emitted from the surface with velocity $v$ to reach the point $(\xi, \eta)$. The boundary condition for the inner equation then reads

$$
\hat{f}(\xi, h(x, \xi), \tau, x, v, t)=M(v) \rho(\xi, \tau, x, t)-\tilde{f}(x, 0, v, t) \quad \text { for } \sigma(x, \xi, v) \leq 0 .
$$

In order to apply the boundary condition to the solution in (2.5), we need to guarantee that $\sigma\left(x, \xi-v_{1} \phi_{0}, v\right) \leq 0$ in the case when the characteristic traces back to the boundary, i.e., $\phi_{0}$ is finite.

Lemma 2.1. If $\phi_{0}(\xi, \eta, x, v)$ is finite and either (i) $\eta=h(x, \xi)$ and $\sigma(x, \xi, v)>0$ or (ii) $\eta>h(x, \xi)$, then it holds that $\sigma\left(x, \xi-v_{1} \phi_{0}, v\right) \leq 0$.

Proof. Define

$$
g(s)=\eta-s v_{2}-h\left(x, \xi-s v_{1}\right)
$$


By definition of $\phi_{0}$, the first positive root of $g(s)$ is given by $s=\phi_{0}$. Then

$$
g^{\prime}(s)=-v_{2}+v_{1} \partial_{\xi} h\left(x, \xi-s v_{1}\right)=\sigma\left(x, \xi-s v_{1}, v\right),
$$

and we have to show that $g^{\prime}\left(\phi_{0}\right)=\sigma\left(x, \xi-v_{1} \phi_{0}, v\right) \leq 0$.

Case (i). If $\eta=h(x, \xi)$ and $\sigma(x, \xi, v)>0$, then

$g(0)=0, \quad g^{\prime}(0)>0 \quad \Rightarrow \quad g(s)>0 \quad$ for $0<s<\phi_{0}(\xi, \eta, x, v) \quad \Rightarrow \quad g^{\prime}\left(\phi_{0}\right) \leq 0$.

Case (ii). If $\eta>h(x, \xi)$, then

$g(0)>0 \Rightarrow g(s)>0 \quad$ for $s<\phi_{0}(\xi, \eta, x, v), \quad g\left(\phi_{0}\right)=0 \quad \Rightarrow \quad g^{\prime}\left(\phi_{0}\right) \leq 0$.

THEOREM 2.2. The solution $\hat{f}(\xi, \eta, \tau, x, v, t)$ to the inner problem (2.4) with boundary condition (2.7) and initial condition $\hat{f}=0$ at $\tau=0$ is given by

$$
\hat{f}(\xi, \eta, \tau, x, v, t)=\left\{\begin{array}{c}
M(v) \rho(\xi, \tau, x, t)-\tilde{f}(x, y=0, v, t) \\
\quad \text { if } \eta=h(x, \xi) \text { and } \sigma(x, \xi, v) \leq 0, \\
H\left(\tau-\phi_{0}\right)\left(M(v) \rho\left(\xi-v_{1} \phi_{0}, \tau-\phi_{0}, x, t\right)-\tilde{f}(x, y=0, v, t)\right) \\
\text { if (i) } \eta=h(x, \xi) \text { and } \sigma(x, \xi, v)>0 \text { or (ii) } \eta>h(x, \xi),
\end{array}\right.
$$

with the intersection time $\phi_{0}$ given by (2.6).

Proof. Define the characteristics

$$
g(s, \xi, \eta, \tau, x, v, t):=\hat{f}\left(\xi-s v_{1}, \eta-s v_{2}, \tau-s, x, v, t\right) ;
$$

then $d g / d s=0$. Follow the characteristics in four possible cases, as follows.

Case 1. If $\eta>h(x, \xi)$ and the line $\left(\xi-s v_{1}, \eta-s v_{2}, \tau-s\right)$ intersects $\tau=0$ first $\left(\tau<\phi_{0}(\xi, \eta, x, v)\right)$, then

$$
\hat{f}(\xi, \eta, \tau, x, v, t)=\hat{f}\left(\xi-\tau v_{1}, \eta-\tau v_{2}, \tau=0, x, v, t\right)=0 .
$$

Case 2. If $\eta>h(x, \xi)$ and the line $\left(\xi-s v_{1}, \eta-s v_{2}, \tau-s\right)$ intersects $\eta=h(x, \xi)$ first $\left(\tau>\phi_{0}(\xi, \eta, x, v)\right)$, then

$$
\hat{f}(\xi, \eta, \tau, x, v, t)=\hat{f}\left(\xi-v_{1} \phi_{0}, \eta-v_{2} \phi_{0}, \tau-\phi_{0}, x, v, t\right), \quad \phi_{0}=\phi_{0}(\xi, \eta, x, v) .
$$

Then $\eta-v_{2} \phi_{0}(\xi, \eta, x, v)=h\left(x, \xi-v_{1} \phi_{0}(\xi, \eta, x, v)\right)$, and hence

$$
\hat{f}(\xi, \eta, \tau, x, v, t)=M(v) \rho\left(\xi-v_{1} \phi_{0}, \tau-\phi_{0}, x, t\right)-\tilde{f}(x, 0, v, t),
$$

because $\sigma\left(x, \xi-\phi_{0} v_{1}, v\right) \leq 0$ by Lemma 2.1 .

Case 3. If $\eta=h(x, \xi)$ and $\sigma(x, \xi, v) \leq 0$ (boundary condition), then

$$
\hat{f}(\xi, h(x, \xi), \tau, x, v, t)=M(v) \rho(\xi, \tau, x, t)-\tilde{f}(x, 0, v, t) .
$$

Case 4. If $\eta=h(x, \xi)$ and $\sigma(x, \xi, v)>0$, then follow the ray $\left(\xi-s v_{1}, \eta-s v_{2}, \tau-s\right)$ back until either $\tau-s=0$ or $\eta-s v_{2}=h\left(x, \xi-s v_{1}\right)$. We distinguish the following two subcases.

(a) If $\eta=h(x, \xi)$ and $\sigma(x, \xi, v)>0$ and $\tau<\phi_{0}(\xi, \eta, x, v)$ (that is, $\tau=0$ is intersected first), then

$$
\hat{f}(\xi, \eta, \tau, x, v, t)=\hat{f}\left(\xi-\tau v_{1}, h-\tau v_{2}, \tau=0, x, v, t\right)=0 .
$$


(b) If $\eta=h(x, \xi)$ and $\sigma(x, \xi, v)>0$ and $\tau>\phi_{0}(\xi, \eta, x, v)$ (that is, $\eta=h(x, \xi)$ is intersected first), then

$$
\begin{gathered}
\hat{f}(\xi, \eta, \tau, x, v, t)=\hat{f}\left(\xi-v_{1} \phi_{0}, h-v_{2} \phi_{0}, \tau-\phi_{0}, x, v, t\right), \\
\phi_{0}=\phi_{0}(\xi, h(x, \xi), x, v),
\end{gathered}
$$

and

$$
\hat{f}(\xi, \eta, \tau, x, v, t)=M(v) \rho\left(\xi-v_{1} \phi_{0}, \tau-\phi_{0}, x, t\right)-\tilde{f}(x, 0, v, t),
$$

because $\sigma\left(x, \xi-\phi_{0} v_{1}, v\right) \leq 0$ by Lemma 2.1 .

We now turn to the evolution of the surface density $\rho$ defined in (2.2). On the one hand, according to (2.2), $\rho$ is defined in terms of $\tilde{f}$ and $\hat{f}$ evaluated at the surface $y=\varepsilon h\left(x, \frac{x}{\varepsilon}\right)$. On the other hand, according to Theorem 2.2, the inner solution $\hat{f}$ at the surface is given in terms of $\rho$ and $\tilde{f}$. Combining these two formulas, we are able to write an evolution equation for $\rho$ in terms of the outer solution $\tilde{f}$ alone. In other words, we are able to write $\rho=F[\tilde{f}]$ with some integral operator to be defined. Moreover, for the computation of the reduced boundary condition (1.13), we will need to make a statement about the behavior of $\rho(\xi, \tau, x, t)$ for the fast time variable $\tau \rightarrow \infty$. To this end, it is convenient to make the following definitions. First we introduce for convenience a new symbol for the function $\phi_{0}$ in Theorem 2.2 evaluated at the boundary by defining $\phi_{1}(\xi, x, v):=\phi_{0}(\xi, h(x, \xi), x, v)$, that is,

$$
\phi_{1}(\xi, x, v)=\left\{\begin{array}{l}
\min \left\{s>0: h(x, \xi)-v_{2} s=h\left(x, \xi-v_{1} s\right)\right\} \\
\infty \quad \text { if } h(x, \xi)-v_{2} s \neq h\left(x, \xi-v_{1} s\right) \text { for all } s>0 .
\end{array}\right.
$$

The function $\phi_{1}$ denotes the time taken by molecules emitted from the boundary to reach another point at the boundary, and is formally set to $\infty$ if they never do. Furthermore, we define the indicator function on the set of all $\xi, x, v$ for which, formally, $\phi_{1}$ is infinite:

$$
\chi(\xi, x, v)= \begin{cases}0 & \text { if } \phi_{1}(\xi, x, v)<\infty \\ 1 & \text { if } \phi_{1}(\xi, x, v)=\infty .\end{cases}
$$

To analyze the limiting behavior of the surface density $\rho$ as $\tau \rightarrow \infty$ we will need that the corresponding limiting problem actually has a solution. This is the statement of the following lemma.

LEMma 2.3. The integral equation

$$
\begin{aligned}
& Z(\xi, x)=g(\xi, x) \\
& +\int H(\sigma(x, \xi, w)) a(x, \xi, w)(1-\chi(\xi, x, w)) M(w) Z\left(\xi-w_{1} \phi_{1}(\xi, x, w), x\right) d w
\end{aligned}
$$

has a solution $Z(\xi, x)$ for any function $g(\xi, x)$, provided that

$$
\int H(\sigma(x, \xi, w)) a(x, \xi, w)(1-\chi(\xi, x, w)) M(w) d w \leq C
$$

for some constant $0 \leq C<1$.

Proof. Introduce the notation

$$
A(x, \xi, w):=H(\sigma(x, \xi, w)) a(x, \xi, w)(1-\chi(\xi, x, w)) M(w),
$$


which satisfies $A(x, \xi) \geq 0$ since $a(x, \xi, w)$ and all other terms are nonnegative. Then we consider the integral equation

$$
Z(\xi, x)=\int A(x, \xi, w) Z\left(\xi-w_{1} \phi_{1}(\xi, x, w), x\right) d w+g(\xi, x)
$$

To compute the solution iteratively, introduce the fixed-point iteration for $\left\{Z_{n}(\xi, x)\right\}$ by

$$
Z_{n+1}(\xi, x)=\int A(x, \xi, w) Z_{n}\left(\xi-w_{1} \phi_{1}(\xi, x, w), x\right) d w+g(\xi, x) \quad \text { for } n=0,1,2, \ldots
$$

with initial iterate $Z_{0}(\xi, x)=0$. Hence, the difference between successive iterates satisfies

$$
\left(Z_{n+1}-Z_{n}\right)(\xi, x)=\int A(x, \xi, w)\left(Z_{n}-Z_{n-1}\right)\left(\xi-w_{1} \phi_{1}(\xi, x, w), x\right) d w,
$$

and we can bound it as

$$
\left\|Z_{n+1}-Z_{n}\right\|_{\infty} \leq \int A(x, \xi, w) d w\left\|Z_{n}-Z_{n-1}\right\|_{\infty} .
$$

The convergence of this sequence is guaranteed if condition (2.10) is satisfied.

Remark 2.1. Condition (2.10) constitutes a restriction on the function $a(x, \xi, w)$ in the boundary condition chosen in the application. It will be verified in section 3 for our choice of $a(x, \xi, w)$.

TheOREM 2.4. If $a(x, \xi, w)$ satisfies (2.10), then the boundary density $\rho$ is of the form

$$
\rho(\xi, \tau, x, t)=F_{\infty}[\tilde{f}](\xi, x, t)+\rho_{1}(\xi, \tau, x, t),
$$

where $F_{\infty}[\tilde{f}]=\rho_{\infty}$ is given implicitly by the integral equation

$$
\begin{aligned}
& \rho_{\infty}(\xi, x, t)=\int H(\sigma(x, \xi, w)) a(x, \xi, w) \chi(\xi, x, w) \tilde{f}(x, 0, w, t) d w \\
& \quad+\int H(\sigma(x, \xi, w)) a(x, \xi, w)(1-\chi(\xi, x, w)) M(w) \rho_{\infty}\left(\xi-w_{1} \phi_{1}, x, t\right) d w,
\end{aligned}
$$

and the remainder term $\rho_{1}$ satisfies $\int_{0}^{\infty} \rho_{1}(\xi, \tau, x, t) d \tau<\infty$.

Proof. Inserting the expression for $\hat{f}(\xi, h(x, \xi), \tau, x, v, t)$ for $\sigma(x, \xi, v)>0$ from Theorem 2.2 into the definition (2.2) for $\rho$ gives

$$
\begin{aligned}
& \rho(\xi, \tau, x, t)=\int H(\sigma(x, \xi, w)) a(x, \xi, w)\left[1-H\left(\tau-\phi_{1}(\xi, x, w)\right)\right] \tilde{f}(x, 0, w, t) d w \\
& \quad+\int H(\sigma(x, \xi, w)) a(x, \xi, w) H\left(\tau-\phi_{1}(\xi, x, w)\right) M(w) \rho\left(\xi-w_{1} \phi_{1}, \tau-\phi_{1}, x, t\right) d w
\end{aligned}
$$

We are interested in the limiting behavior for $\tau \rightarrow \infty$. To this end, we have to distinguish the cases when $\phi_{1}<\infty$ holds, which means that the Heaviside function in (2.12) will become equal to unity for $\tau$ sufficiently large, and the case $\phi_{1}=\infty$ for 
which the Heaviside function will always be zero. Therefore we write

$$
\begin{aligned}
& \rho(\xi, \tau, x, t) \\
& =\int H(\sigma(x, \xi, w)) a(x, \xi, w)\left[1-(1-\chi(\xi, x, w)) H\left(\tau-\phi_{1}(\xi, x, w)\right)\right] \tilde{f}(x, 0, w, t) d w \\
& +\int H(\sigma(x, \xi, w)) a(x, \xi, w)(1-\chi(\xi, x, w)) H\left(\tau-\phi_{1}(\xi, x, w)\right) M(w) \\
& \quad \times \rho\left(\xi-w_{1} \phi_{1}, \tau-\phi_{1}, x, t\right) d w,
\end{aligned}
$$

with $\chi$ defined as in (2.9). Letting, formally, $\tau$ tend to infinity gives $\rho=\rho_{\infty}+\rho_{1}$ with (2.11) for $\rho_{\infty}(\xi, x, t)$. The solution $\rho_{\infty}$ exists due to Lemma 2.3. This is, of course, only a formal definition for $\rho_{\infty}$; the key is to estimate the remainder term $\rho_{1}$. The remainder term $\rho_{1}$ satisfies the integral equation

$$
\begin{aligned}
& \rho_{1}(\xi, \tau, x, t) \\
& =\int H(\sigma(x, \xi, w)) a(x, \xi, w)(1-\chi(\xi, x, w)) H\left(\tau-\phi_{1}(\xi, x, w)\right) M(w) \\
& \quad \times \rho_{1}\left(\xi-w_{1} \phi_{1}, \tau-\phi_{1}, x, t\right) d w \\
& +\int H(\sigma(x, \xi, w)) a(x, \xi, w)(1-\chi(\xi, x, w))\left(1-H\left(\tau-\phi_{1}(\xi, x, w)\right)\right) \\
& \quad \times\left(\tilde{f}(x, 0, w, t)-M(w) \rho_{\infty}\left(\xi-w_{1} \phi_{1}, x, t\right)\right) d w .
\end{aligned}
$$

Let $\mathcal{L}\left[\rho_{1}\right](\xi, s, x, t):=\int_{0}^{\infty} \rho_{1}(\xi, \tau, x, t) e^{-s \tau} d \tau$, with $0 \leq s<\infty$, denote the Laplace transform of $\rho_{1}$. The transform of (2.14) is then given by

$$
\begin{aligned}
& \mathcal{L}\left[\rho_{1}\right](\xi, s, x, t) \\
& =\int H(\sigma(x, \xi, w)) a(x, \xi, w)(1-\chi(\xi, x, w)) M(w) e^{-s \phi_{1}(x, \xi, w)} \\
& \quad \times \mathcal{L}\left[\rho_{1}\right]\left(\xi-w_{1} \phi_{1}, s, x, t\right) d w \\
& +\int H(\sigma(x, \xi, w)) a(x, \xi, w)(1-\chi(\xi, x, w)) \frac{1}{s}\left[1-e^{-s \phi_{1}(x, \xi, w)}\right] \\
& \quad \times\left(\tilde{f}(x, 0, w, t)-M(w) \rho_{\infty}\left(\xi-w_{1} \phi_{1}, x, t\right)\right) d w .
\end{aligned}
$$

The variable $s$ appears as a parameter only in the integral equation, so in order to find $\mathcal{L}\left[\rho_{1}\right](\xi, 0, x, t)$, we can solve directly

$$
\begin{aligned}
& \mathcal{L}\left[\rho_{1}\right](\xi, 0, x, t) \\
& =\int H(\sigma(x, \xi, w)) a(x, \xi, w)(1-\chi(\xi, x, w)) M(w) \mathcal{L}\left[\rho_{1}\right]\left(\xi-w_{1} \phi_{1}, 0, x, t\right) d w \\
& +\int H(\sigma(x, \xi, w)) a(x, \xi, w)(1-\chi(\xi, x, w)) \frac{1}{\phi_{1}} \\
& \quad \times\left(\tilde{f}(x, 0, w, t)-M(w) \rho_{\infty}\left(\xi-w_{1} \phi_{1}, x, t\right)\right) d w .
\end{aligned}
$$

By Lemma 2.3, the solution $\mathcal{L}\left[\rho_{1}\right](\xi, 0, x, t)$ exists, and we therefore have

$$
\int_{0}^{\infty} \rho_{1}(\xi, \tau, x, t) d \tau=\mathcal{L}\left[\rho_{1}\right](\xi, 0, x, t)<\infty
$$

by definition of the transform. 
Remark 2.2. Theorem 2.4 establishes the limiting behavior of $\rho$ for $\tau \rightarrow \infty$ in a weak sense, namely, that $\rho_{1}\left(\xi, \frac{t}{\varepsilon}, x, t\right)$ will go to zero when integrated with respect to $t$ against a test function. This is precisely the property that will be needed for the derivation of the boundary condition.

2.2. Boundary condition for the outer solution. This subsection will use the weak convergence property for the inner solution to derive the boundary condition for the outer solution. Because the layer equation (2.4) does not have any damping, $\hat{f}$ will give a contribution throughout the half-space $y>0$. However, this contribution will result in high frequency oscillations only for $y>0$. Therefore, we formulate the Boltzmann equation in the weak sense and require that $\hat{f}$ tend to zero weakly for fixed $y>0$ and $\varepsilon \rightarrow 0$. Thus, we require in a weak sense for $y, v$ fixed that

$$
\lim _{\varepsilon \rightarrow 0} \int_{0}^{\infty} \int_{-\infty}^{+\infty} \hat{f}\left(\frac{x}{\varepsilon}, \frac{y}{\varepsilon}, \frac{t}{\varepsilon}, x, v, t\right) \psi(x, t) d x d t=0
$$

for all test functions $\psi(x, t)$.

THEOREM 2.5. The integral in (2.16) goes to zero for $\varepsilon \rightarrow 0$ and all fixed $y>0$, $v$ if and only if

$$
\tilde{f}(x, 0, v, t)=M(v) \int_{0}^{1} F_{\infty}[\tilde{f}]\left(\xi-v_{1} \phi_{3}, x, t\right) d \xi \quad \text { for all } v_{2}>0
$$

holds with

$$
\phi_{3}(\xi, x, v)=\min \left\{s \in \mathbb{R}:-v_{2} s=h\left(x, \xi-v_{1} s\right)\right\}
$$

and the operator $F_{\infty}[\tilde{f}]$ defined as in Theorem 2.4.

Proof. Let $I_{\varepsilon}:=\iint \hat{f}\left(\frac{x}{\varepsilon}, \frac{y}{\varepsilon}, \frac{t}{\varepsilon}, x, v, t\right) \psi(x, t) d x d t$ denote the integral in (2.16). We need to estimate $I_{\varepsilon}$ for velocities $v$ with $v_{2}>\kappa(\varepsilon)$, where $\kappa$ is some function with $\kappa(\varepsilon)>0$ and $\kappa(\varepsilon) \rightarrow 0$ as well as $\varepsilon / \kappa(\varepsilon) \rightarrow 0$ as $\varepsilon \rightarrow 0$. Since we are interested in the bulk solution $\tilde{f}(x, y, v, t)$, we need to consider only $\eta=\frac{y}{\varepsilon}>h\left(x, \frac{x}{\varepsilon}\right)$.

We have from Theorem 2.2 for $\frac{y}{\varepsilon}>h\left(x, \frac{x}{\varepsilon}\right)$ and $v_{2}>\kappa(\varepsilon)>0$

$$
\hat{f}\left(\frac{x}{\varepsilon}, \frac{y}{\varepsilon}, \frac{t}{\varepsilon}, x, v, t\right)=H\left(\frac{t}{\varepsilon}-\phi_{0}\right)\left(M(v) \rho\left(\frac{x}{\varepsilon}-v_{1} \phi_{0}, \frac{t}{\varepsilon}-\phi_{0}, x, t\right)-\tilde{f}(x, 0, v, t)\right),
$$

with $\phi_{0}\left(\frac{x}{\varepsilon}, \frac{y}{\varepsilon}, x, v\right)=\min \left\{s>0: \frac{y}{\varepsilon}-v_{2} s=h\left(x, \frac{x}{\varepsilon}-v_{1} s\right)\right\}$, where this simplified (compared to (2.6)) definition for $\phi_{0}$ is possible because the existence of the minimum is guaranteed for $v_{2}>\kappa(\varepsilon)>0$. To transform

$$
\phi_{0}\left(\frac{x}{\varepsilon}, \frac{y}{\varepsilon}, x, v\right)=\frac{y}{v_{2} \varepsilon}+\phi_{2}\left(\frac{x}{\varepsilon}-\frac{v_{1} y}{v_{2} \varepsilon}, \frac{y}{\varepsilon}, x, v\right),
$$

define

$$
\phi_{2}\left(\frac{x}{\varepsilon}-\frac{v_{1} y}{v_{2} \varepsilon}, \frac{y}{\varepsilon}, x, v\right)=\min \left\{s>-\frac{y}{v_{2} \varepsilon}:-v_{2} s=h\left(x, \frac{x}{\varepsilon}-\frac{v_{1} y}{v_{2} \varepsilon}-v_{1} s\right)\right\} .
$$

This gives

$$
\begin{aligned}
& \hat{f}\left(\frac{x}{\varepsilon}, \frac{y}{\varepsilon}, \frac{t}{\varepsilon}, x, v, t\right) \\
& =H\left(\frac{t}{\varepsilon}-\frac{y}{v_{2} \varepsilon}-\phi_{2}\right)\left(M(v) \rho\left(\frac{x}{\varepsilon}-\frac{v_{1} y}{v_{2} \varepsilon}-v_{1} \phi_{2}, \frac{t}{\varepsilon}-\frac{y}{v_{2} \varepsilon}-\phi_{2}, x, t\right)-\tilde{f}(x, 0, v, t)\right),
\end{aligned}
$$


with $\phi_{2} \equiv \phi_{2}\left(\frac{x}{\varepsilon}-\frac{v_{1} y}{v_{2} \varepsilon}, \frac{y}{\varepsilon}, x, v\right)$ for $v_{2}>\kappa(\varepsilon)$.

We introduce the transformation

$$
x=x_{j \xi}:=\frac{v_{1}}{v_{2}} y+\varepsilon j+\varepsilon \xi, \quad j \in \mathbb{Z}, \quad \xi \in[0,1],
$$

to rewrite $I_{\varepsilon}$ into

$$
\begin{gathered}
I_{\varepsilon}=\varepsilon \sum_{j=-\infty}^{+\infty} \int_{0}^{\infty} \int_{0}^{1} H\left(\frac{t}{\varepsilon}-\frac{y}{v_{2} \varepsilon}-\phi_{2}\right)\left(M(v) \rho\left(j+\xi-v_{1} \phi_{2}, \frac{t}{\varepsilon}-\frac{y}{v_{2} \varepsilon}-\phi_{2}, x_{j \xi}, t\right)\right. \\
\left.-\tilde{f}\left(x_{j \xi}, 0, v, t\right)\right) \psi\left(x_{j \xi}, t\right) d \xi d t
\end{gathered}
$$

with $\phi_{2} \equiv \phi_{2}\left(j+\xi, \frac{y}{\varepsilon}, x_{j \xi}, v\right)=\min \left\{s>-\frac{y}{v_{2} \varepsilon}:-v_{2} s=h\left(x_{j \xi}, j+\xi-v_{1} s\right)\right\}$. Because $h(x, \xi)$ and hence also $\rho$ and $\phi_{2}$ are 1-periodic in $\xi$, we can drop the $j$ from their first arguments. Since $\rho, \tilde{f}$, and $\psi$ vary only slowly in $x$ and since $\xi$ varies only in $[0,1]$, we make an $\mathcal{O}(\varepsilon)$ perturbation by replacing $x_{j \xi}$ by $x_{j}:=\frac{v_{1}}{v_{2}} y+\varepsilon j$. Together this gives

$$
\begin{array}{r}
I_{\varepsilon}=\varepsilon \sum_{j=-\infty}^{+\infty} \int_{0}^{\infty} \int_{0}^{1} H\left(\frac{t}{\varepsilon}-\frac{y}{v_{2} \varepsilon}-\phi_{2}\right)\left(M(v) \rho\left(\xi-v_{1} \phi_{2}, \frac{t}{\varepsilon}-\frac{y}{v_{2} \varepsilon}-\phi_{2}, x_{j}, t\right)\right. \\
\left.-\tilde{f}\left(x_{j}, 0, v, t\right)\right) \psi\left(x_{j}, t\right) d \xi d t+\mathcal{O}(\varepsilon)
\end{array}
$$

with $\phi_{2} \equiv \phi_{2}\left(\xi, \frac{y}{\varepsilon}, x_{j}, v\right)=\min \left\{s>-\frac{y}{v_{2} \varepsilon}:-v_{2} s=h\left(x_{j}, \xi-v_{1} s\right)\right\}$. The sum over the $j$ forms a Riemann sum for an integral with approximation error $\mathcal{O}(\varepsilon)$. Thus, we obtain

$$
\begin{array}{r}
I_{\varepsilon}=\int_{0}^{\infty} \int_{-\infty}^{+\infty} \int_{0}^{1} H\left(\frac{t}{\varepsilon}-\frac{y}{v_{2} \varepsilon}-\phi_{2}\right)\left(M(v) \rho\left(\xi-v_{1} \phi_{2}, \frac{t}{\varepsilon}-\frac{y}{v_{2} \varepsilon}-\phi_{2}, z, t\right)\right. \\
-\tilde{f}(z, 0, v, t)) \psi(z, t) d \xi d z d t+\mathcal{O}(\varepsilon),
\end{array}
$$

with $\phi_{2} \equiv \phi_{2}\left(\xi, \frac{y}{\varepsilon}, z, v\right)=\min \left\{s>-\frac{y}{v_{2} \varepsilon}:-v_{2} s=h\left(z, \xi-v_{1} s\right)\right\}$.

Now we replace $\rho=\rho_{\infty}+\rho_{1}$ with $F_{\infty}[\tilde{f}]=\rho_{\infty}$, as defined in Theorem 2.4. For the remainder term involving $\rho_{1}$ this gives

$$
\begin{aligned}
E_{\varepsilon}:=\int_{0}^{\infty} \int_{-\infty}^{+\infty} \int_{0}^{1} H\left(\frac{t}{\varepsilon}-\frac{y}{v_{2} \varepsilon}-\phi_{2}\right) M(v) \rho_{1}(\xi & \left.-v_{1} \phi_{2}, \frac{t}{\varepsilon}-\frac{y}{v_{2} \varepsilon}-\phi_{2}, z, t\right) \\
& \times \psi(z, t) d \xi d z d t
\end{aligned}
$$

We transform again to a fast variable $\tau:=\frac{t}{\varepsilon}-\frac{y}{v_{2} \varepsilon}-\phi_{2}$ and obtain

$$
\begin{array}{r}
E_{\varepsilon}=\varepsilon M(v) \int_{0}^{\infty} \int_{-\infty}^{+\infty} \int_{0}^{1} \rho_{1}\left(\xi-v_{1} \phi_{2}, \tau, z, \frac{y}{v_{2}}+\varepsilon \tau+\varepsilon \phi_{2}\right) \\
\times \psi\left(z, \frac{y}{v_{2}}+\varepsilon \tau+\varepsilon \phi_{2}\right) d \xi d z d \tau .
\end{array}
$$

Since the test function $\psi$ is smooth and has compact support, and since Theorem 2.4 guarantees that $\int_{0}^{\infty} \rho_{1}\left(\xi-v_{1} \phi_{2}, \tau, z, t\right) d \tau$ is bounded, the integrals in $E_{\varepsilon}$ remain bounded, and $E_{\varepsilon} \rightarrow 0$, as $\varepsilon \rightarrow 0$. 
In the remaining parts of (2.18), we use the fact that the Heaviside function is scaling-invariant to get $H\left(\frac{t}{\varepsilon}-\frac{y}{v_{2} \varepsilon}-\phi_{2}\right)=H\left(t-\frac{y}{v_{2}}-\varepsilon \phi_{2}\right)$. From the definition (2.17) of $\phi_{2}$ it follows that, for $v_{2}>\kappa(\varepsilon)>0,\left|\phi_{2}\right| \leq \max \{h\} / \kappa(\varepsilon)$ holds. Therefore, the dependence of the Heaviside function on $\varepsilon \phi_{2}$ is negligible, and it can be taken out of the $\xi$-integral, introducing only another $\mathcal{O}\left(\frac{\varepsilon}{\kappa}\right)$ error. Also, the integrand involving $\tilde{f}$ does not depend on the fast variable $\xi$ any more, and so the integration over the interval $[0,1]$ yields just unity. This gives after $\varepsilon \rightarrow 0$

$$
0=\int_{0}^{\infty} \int_{-\infty}^{+\infty} H\left(t-\frac{y}{v_{2}}\right)\left(M(v) \int_{0}^{1} \rho_{\infty}\left(\xi-v_{1} \phi_{2}, z, t\right) d \xi-\tilde{f}(z, 0, v, t)\right) \psi(z, t) d z d t
$$

for any fixed $v_{2}>0$. Taking the limit $\varepsilon \rightarrow 0$ in $\phi_{2} \equiv \phi_{2}\left(\xi, \frac{y}{\varepsilon}, z, v\right)=\min \left\{s>-\frac{y}{v_{2} \varepsilon}\right.$ : $\left.-v_{2} s=h\left(z, \xi-v_{1} s\right)\right\}$ results in the minimum being taken over the entire real line, since $y>0$ and $v_{2}>0$. It also makes $\phi_{2}$ independent of $\varepsilon$ and $y$, and we introduce the notation

$$
\phi_{3} \equiv \phi_{3}(\xi, z, v)=\min \left\{s \in \mathbb{R}:-v_{2} s=h\left(z, \xi-v_{1} s\right)\right\} .
$$

Therefore, (2.19) is satisfied in a weak sense if and only if

$$
\tilde{f}(z, 0, v, t)=M(v) \int_{0}^{1} \rho_{\infty}\left(\xi-v_{1} \phi_{3}, z, t\right) d \xi
$$

holds for all $z, v, t$ with $v_{2}>0$.

Remark 2.3. For any fixed velocity $v$ with $v_{2}>0$, the function $\phi_{3}$ is guaranteed to exist with $-\max \{h(x, \xi)\} / v_{2} \leq \phi_{3}(\xi, x, v) \leq-\min \{h(x, \xi)\} / v_{2}$, since the surface function $h(x, \xi)$ is smooth.

Theorem 2.5 essentially yields the reduced boundary condition. In practice, one will not solve the integral equation $(2.11)$ to compute $F_{\infty}[\tilde{f}]$ at every time step. It is preferable to write the term $F_{\infty}[\tilde{f}]$ as an integral operator with a time independent integral kernel. A direct calculation leads from (2.11) to

$$
F_{\infty}[\tilde{f}](\xi, x, t)=\int K_{\infty}(\xi, x, v) \tilde{f}(x, 0, v, t) d v
$$

where the integral kernel $K_{\infty}$ satisfies

$$
\begin{aligned}
& K_{\infty}(\xi, x, v)=H(\sigma(x, \xi, v)) a(x, \xi, v) \chi(\xi, x, v) \\
& \quad+\int H(\sigma(x, \xi, w)) a(x, \xi, w)(1-\chi(\xi, x, w)) M(w) K_{\infty}\left(\xi-w_{1} \phi_{1}(\xi, x, w), x, v\right) d w
\end{aligned}
$$

and $K_{\infty}(\xi, x, v)$ again exists because of Lemma 2.3. Note that $K_{\infty}$ will be nonnegative, provided that $a(x, \xi, w)$ is not too large.

In summary, we have obtained the following numerical problem for the bulk solution $\tilde{f}(x, y, v, t)$ :

$$
\frac{\partial \tilde{f}}{\partial t}+v_{1} \frac{\partial \tilde{f}}{\partial x}+v_{2} \frac{\partial \tilde{f}}{\partial y}=Q(\tilde{f})
$$


with boundary condition for inflowing molecules on the flat surface $y=0$

$$
\tilde{f}(x, 0, v, t)=M(v) \int \tilde{a}(x, v, w) \tilde{f}(x, 0, w, t) d w \quad \text { for } v_{2}>0,
$$

with

$$
\tilde{a}(x, v, w):=\int_{0}^{1} K_{\infty}\left(\xi-v_{1} \phi_{3}(\xi, x, v), x, w\right) d \xi
$$

and

$$
\phi_{3}(\xi, x, v)=\min \left\{s \in \mathbb{R}:-v_{2} s=h\left(x, \xi-v_{1} s\right)\right\} .
$$

This problem is tractable numerically, since it is posed on a domain with a flat reacting surface; the effect of the microscopic surface has been integrated into the boundary condition. The numerical approach in practice will be as follows.

Step 1. Given a surface function $h(x, \xi)$, compute the intersection times $\phi_{1}$ in $(2.8)$ and $\phi_{3}$ in (2.24).

Step 2. Solve the integral equation $(2.20)$ for $K_{\infty}$.

Step 3. Compute the boundary kernel $\tilde{a}(x, v, w)$ in $(2.23)$.

The function $\tilde{a}(x, v, w)$ provides the information about the microscopic surface geometry on the macroscopic level. These steps have to be performed only once for a given surface function $h(x, \xi)$. Following these preprocessing steps, the Boltzmann equation (2.21) with the homogenized boundary condition (2.22) is solved for the bulk solution $\tilde{f}(x, y, v, t)$.

3. Numerical validation. As a validation problem, we consider the linear Boltzmann equation for a single species

$$
\frac{\partial f}{\partial t}+v_{1} \frac{\partial f}{\partial x}+v_{2} \frac{\partial f}{\partial y}=Q(f)
$$

We use a relaxation time approximation $S\left(v, v^{\prime}\right)=(1 / \tau) M(v) M\left(v^{\prime}\right)$ in the linear collision operator (1.6) to obtain the simple form

$$
Q(f)(x, y, v, t)=-\frac{1}{\tau}[f(x, y, v, t)-N(x, y, t) M(v)]
$$

with the constant relaxation time $\tau>0$. Here and in the following, $N$ denotes the number density given by $N(x, y, t)=\int f(x, y, v, t) d v$. The numerical domain is chosen as

$$
\Omega_{\varepsilon}=\left\{(x, y) \in \mathbb{R}^{2}: \tilde{h}(x)<y<1, \quad 0<x<1\right\},
$$

with a microstructured surface $\Gamma_{\varepsilon}$ at the bottom given by

$$
y=\tilde{h}(x)=\frac{\varepsilon}{8}\left(1+\cos \left(2 \pi \frac{x}{\varepsilon}\right)\right)
$$

with homogenization parameter $0<\varepsilon \ll 1$. This model is designed to closely resemble the salient features of the application problem that motivated the model and to support the above analysis; this motivates also the choice of the simple form of the collision operator. Other types of boundary models, e.g., using random reflections [1], could be used but would not be appropriate examples for our application. 
The following model is chosen as the boundary condition describing the reactions of the gaseous species at the wafer surface that result in the deposition of the solid film. If $0 \leq R \leq 1$ denotes the sticking factor (the probability that a molecule sticks to the surface), then the inflow into the gaseous domain is equal to $(1-R)$ times the outflow from the gaseous domain, namely (see [4]),

$$
\int_{n \cdot v<0}|n \cdot v| f(x, y, v, t) d v=(1-R) \int_{n \cdot v>0}|n \cdot v| f(x, y, v, t) d v,
$$

where $n=n(x, y)$ denotes the unit outward normal vector at position $(x, y) \in \Gamma_{\varepsilon}$. To obtain the boundary condition in the form (1.10), assume reinjection with random velocities, that is, $f(x, y, v, t)=b(x, t) M(v)$ for $n \cdot v<0$; then

$$
f(x, y, v, t)=M(v) \int_{n \cdot w>0} a\left(x, \frac{x}{\varepsilon}, w\right) f(x, w, t) d w,
$$

with

$$
a\left(x, \frac{x}{\varepsilon}, w\right)=\frac{1-R}{c}|n \cdot w|, \quad c=\int_{n \cdot v<0}|n \cdot v| M(v) d v .
$$

The problem is completed by choosing Maxwellian inflow at the top and periodic boundary conditions at both sides. This chosen setup of the problem is representative of the application under consideration. We choose $\tau=1$ and $R=0.5$ as values.

To check condition (2.10) for this choice of $a\left(x, \frac{x}{\varepsilon}, w\right)$, we compute

$$
\begin{aligned}
I\left(x, \frac{x}{\varepsilon}\right) & :=\int H\left(\sigma\left(x, \frac{x}{\varepsilon}, w\right)\right) a\left(x, \frac{x}{\varepsilon}, w\right)\left(1-\chi\left(\frac{x}{\varepsilon}, x, w\right)\right) M(w) d w \\
& =(1-R) \frac{\int_{n \cdot w>0}|n \cdot w|\left(1-\chi\left(\frac{x}{\varepsilon}, x, w\right)\right) M(w) d w}{\int_{n \cdot v<0}|n \cdot v| M(v) d v} .
\end{aligned}
$$

Since $0 \leq \chi \leq 1$, the fraction is always bounded by 1 , which is seen by using the transformation $w=-v$ in the denominator and using the symmetry of the Maxwellian in (1.4). In the case that $0<R \leq 1$, we have $1-R<1$ and $I<1$. In the limiting case $R=0$, we can still conclude that $I<1$, because $\chi$ does not vanish almost everywhere in reasonable situations in the application, and hence the fraction will be less than 1 except in pathological cases.

The problem was solved numerically by choosing an expansion in velocity space of the form following $[14,16,19,20,21,22]$ and the references therein,

$$
f(x, y, v, t)=\sum_{k=1}^{K} f_{k}(x, y, t) \varphi_{k}(v),
$$

where the $\left\{\varphi_{k}(v), k=1, \ldots, K\right\}$ form an orthogonal set of basis functions with respect to the inner product

$$
\left\langle\varphi_{k}, \varphi_{\ell}\right\rangle=\int \varphi_{k}(v) \varphi_{\ell}(v) \omega(v) d v=\delta_{k \ell}
$$

with weight function $\omega(v)=1 / M(v)$. The basis functions are chosen as Maxwellians multiplied by (properly transformed) Hermite polynomials. To arrive at a Gaussian quadrature for the integrals, the discretization points in velocity space are roots of appropriate Hermite polynomials. 
A Galerkin discretization by inserting the expansion for $f$ and forming inner products with all basis functions then leads to the system of hyperbolic equations for the expansion coefficients $F=\left(f_{k}\right)$

$$
\frac{\partial F}{\partial t}+A^{(1)} \frac{\partial F}{\partial x}+A^{(2)} \frac{\partial F}{\partial y}=C F,
$$

with the $K \times K$ matrices $A^{(1)}, A^{(2)}$, and $C$ with components

$$
A_{k, \ell}^{(1)}=\left\langle v_{1} \varphi_{\ell}, \varphi_{k}\right\rangle, \quad A_{k, \ell}^{(2)}=\left\langle v_{2} \varphi_{\ell}, \varphi_{k}\right\rangle, \quad C_{k, \ell}=\left\langle Q\left(\varphi_{\ell}\right), \varphi_{k}\right\rangle .
$$

We actually use an equivalent collocation basis for the Hermite polynomials, which results in the matrices $A^{(1)}$ and $A^{(2)}$ being diagonal; see $[10,13,23]$ for more details. This system is solved by a finite-difference method using first-order upwinding and explicit time-stepping. The solution on the homogenized domain

$$
\Omega_{0}=(0,1) \times(0,1)
$$

is straightforward (that was the point of the homogenization). The comparison solution on the microstructured domain $\Omega_{\varepsilon}$ is obtained by transforming the domain to the unit square. The transformation is designed such that it is an identity in the upper half of the domain, i.e., for $y \geq 0.5$; this is done in order to facilitate the comparison of both numerical solutions there without incurring additional interpolation error from a mesh transformation.

We discretize the velocity space by six basis functions in both $x$ - and $y$-directions, resulting in a hyperbolic system of 36 equations. The maximum velocity is bounded by 4 in each direction. Using $\Delta x=1 / 128$ and $\Delta y=1 / 64$, the CFL condition requires a time step of $\Delta t=1 / 1024$, accounting for an additional factor of about 4 from the transformation of the domain. Solutions are computed until the final time $t_{\text {fin }}=2$. We compute solutions $f(x, y, v, t)$ with number densities denoted by $N(x, y, t)$ on domains $\Omega_{\varepsilon}$ in (3.2), $\varepsilon=1 / 4,1 / 8,1 / 16$, and $1 / 32$. The solution to the homogenized problem is computed on $\Omega_{0}$ in (3.4) and denoted by $\tilde{f}(x, y, v, t)$, with number density $\tilde{N}(x, y, t)$. Smaller values do not yield reliable solutions for the grid spacing used in the $x$-direction.

Figures 3.1, 3.2, 3.3, and 3.4 show comparisons of the number density $\tilde{N}(x, y, t)$ at $T=t_{\text {fin }}=2$ on the homogenized domain to the number density $N(x, y, t)$ on the microstructured domain with the indicated values of $\varepsilon$. The plots show the physical behavior of the flow: The reacting chemical is supplied at the top of the domain at $y=$ 1 , then moves towards the wafer surface at $y=\tilde{h}(x)$, where it gets partially consumed in the surface reaction. Notice that the oscillations of the solution are limited to a boundary layer close to the microstructured surface; this effect is attributable to the smoothing property of the collision operator; that is, the solution is smoother than could be theoretically expected.

Table 3.1 shows the errors between the densities $\tilde{N}(x, y, t)$ and $N(x, y, t)$ of the homogenized and the original problems for various values of $\varepsilon$. They are compared only across the upper half of the domain, i.e., for $y \geq 0.5$, by choosing the subdomain $\tilde{\Omega}=(0,1) \times(0.5,1)$ in the norms

$$
\|N\|_{L^{1}(\tilde{\Omega})}:=\iint_{\tilde{\Omega}}|N(x, y, t)| d x d y
$$

and

$$
\|N\|_{L \infty(\tilde{\Omega})}:=\max _{(x, y) \in \tilde{\Omega}}|N(x, y, t)|
$$




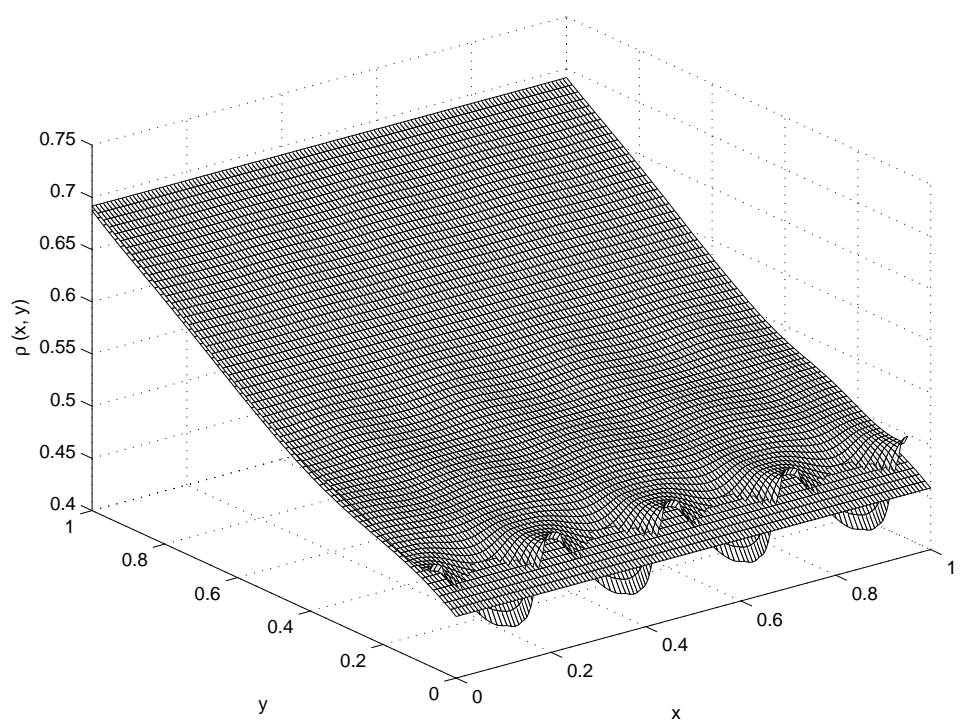

FIG. 3.1. Comparison of the number densities on the homogenized and the microstructured domains with $\varepsilon=1 / 4$.

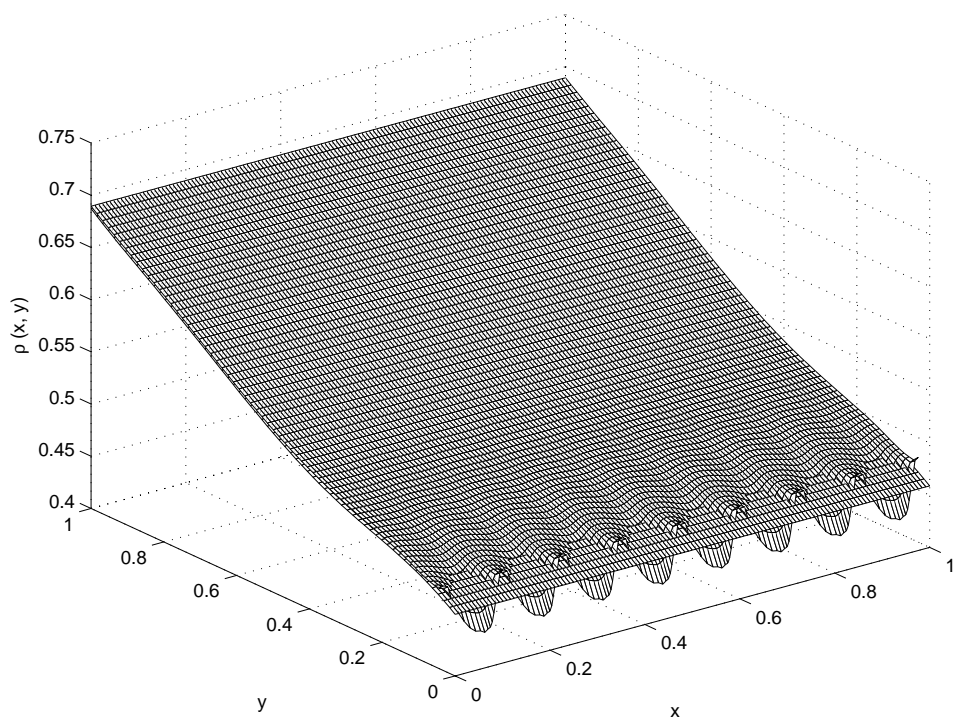

FIG. 3.2. Comparison of the number densities on the homogenized and the microstructured domains with $\varepsilon=1 / 8$.

for a fixed time $t$. The table shows results at the final time $T=t_{f i n}=2$. Notice the decrease of all absolute as well as relative errors with $\varepsilon$.

Tables 3.2 and 3.3 study the underlying error in the density function $f$ itself. More precisely, if $\tilde{f}(x, y, v, t)$ denotes the homogenized solution and $f(x, y, v, t)$ the solution 


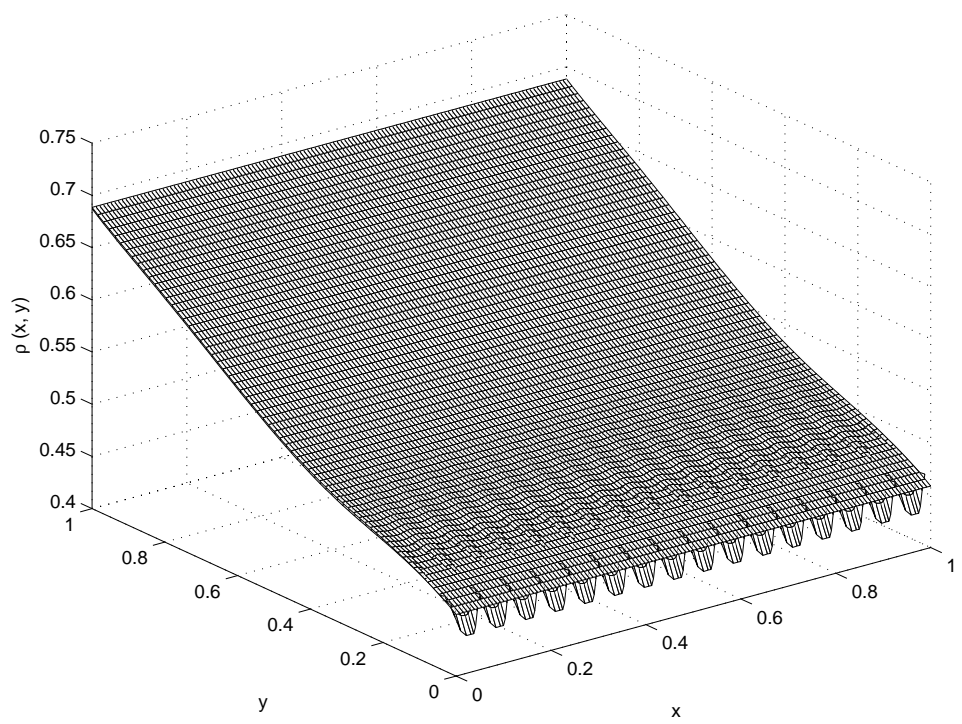

FIG. 3.3. Comparison of the number densities on the homogenized and the microstructured domains with $\varepsilon=1 / 16$.

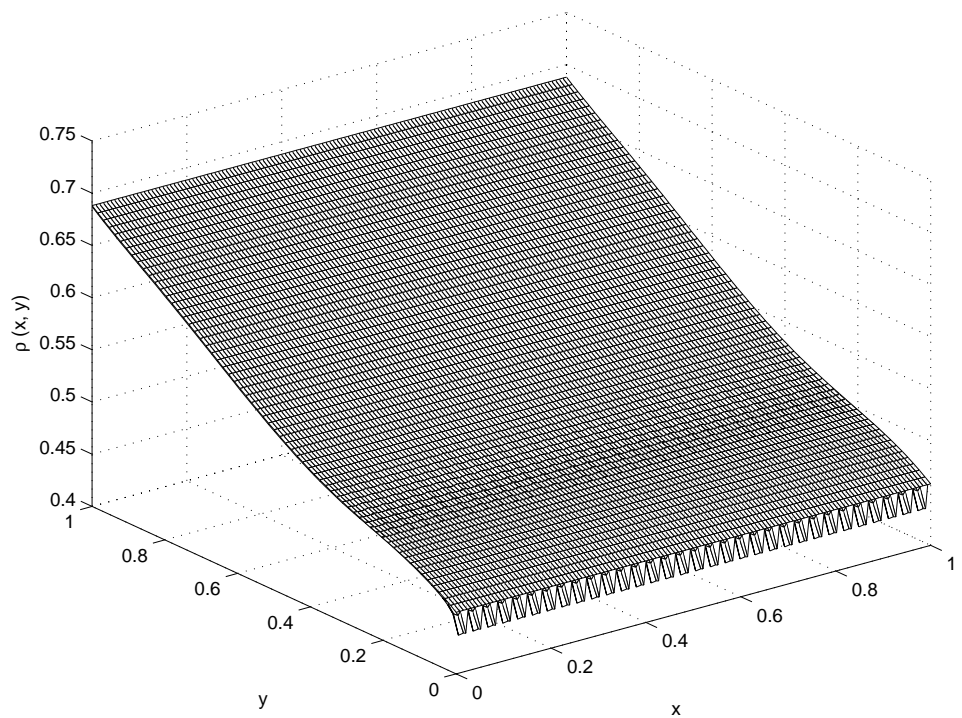

FIG. 3.4. Comparison of the number densities on the homogenized and the microstructured domains with $\varepsilon=1 / 32$.

on the microstructured domain, then Table 3.2 lists the quantity $I(\tilde{f}-f)(y, v, T)$ with

$$
I(f)(y, v, T):=\int_{0}^{T} \int_{0}^{1} f(x, y, v, t) d x d t
$$

with $T=t_{\text {fin }}=2$ at $y=0.875$ (close to the top of the domain) at various points 
TABLE 3.1

Errors in density $\tilde{N}$ measured in various norms $\|\tilde{N}-N\|_{L^{1}(\tilde{\Omega})},\|\tilde{N}-N\|_{L^{1}(\tilde{\Omega})} /\|N\|_{L^{1}(\tilde{\Omega})}$, $\|\tilde{N}-N\|_{L^{\infty}(\tilde{\Omega})}$, and $\|\tilde{N}-N\|_{L^{\infty}(\tilde{\Omega})} /\|N\|_{L_{(\tilde{\Omega})}}$ on subdomain $\tilde{\Omega}=(0,1) \times(0.5,1)$ at $T=t_{f i n}=2$.

\begin{tabular}{lcccc}
\hline & $\|\tilde{N}-N\|_{L^{1}}$ & $\|\tilde{N}-N\|_{L^{1}} /\|N\|_{L^{1}}$ & $\|\tilde{N}-N\|_{L^{\infty}}$ & $\|\tilde{N}-N\|_{L^{\infty}} /\|N\|_{L^{\infty}}$ \\
\hline$\varepsilon=1 / 4$ & 0.0023932 & 0.0076664 & 0.0070134 & 0.0101409 \\
$\varepsilon=1 / 8$ & 0.0013703 & 0.0044039 & 0.0031563 & 0.0045777 \\
$\varepsilon=1 / 16$ & 0.0008171 & 0.0026308 & 0.0017929 & 0.0026041 \\
$\varepsilon=1 / 32$ & 0.0005755 & 0.0018544 & 0.0012608 & 0.0018324 \\
\hline
\end{tabular}

TABLE 3.2

Quantity $I(\tilde{f}-f)(y, v, T)$ with $T=t_{f i n}=2$ at $y=0.875$ and velocity with $v_{1}=-0.6167$ and $v_{2}$ as listed.

\begin{tabular}{lcccccc}
\hline$v_{2}$ & -3.3243 & -1.8892 & -0.6167 & 0.6167 & 1.8892 & 3.3243 \\
\hline$\varepsilon=1 / 4$ & $6.8068 \mathrm{e}-08$ & $4.7740 \mathrm{e}-06$ & $5.6442 \mathrm{e}-05$ & $7.2642 \mathrm{e}-04$ & $3.8061 \mathrm{e}-04$ & $1.1459 \mathrm{e}-05$ \\
$\varepsilon=1 / 8$ & $3.7021 \mathrm{e}-08$ & $2.5996 \mathrm{e}-06$ & $3.0958 \mathrm{e}-05$ & $3.7582 \mathrm{e}-04$ & $2.1973 \mathrm{e}-04$ & $6.4414 \mathrm{e}-06$ \\
$\varepsilon=1 / 16$ & $2.3066 \mathrm{e}-08$ & $1.6244 \mathrm{e}-06$ & $1.9571 \mathrm{e}-05$ & $2.2059 \mathrm{e}-04$ & $1.4038 \mathrm{e}-04$ & $3.6978 \mathrm{e}-06$ \\
$\varepsilon=1 / 32$ & $1.8086 \mathrm{e}-08$ & $1.2793 \mathrm{e}-06$ & $1.5657 \mathrm{e}-05$ & $1.5784 \mathrm{e}-04$ & $1.1535 \mathrm{e}-04$ & $2.7049 \mathrm{e}-06$ \\
\hline
\end{tabular}

TABLE 3.3

Quantity $I(\tilde{f}-f)(y, v, T) / I(f)(y, v, T)$ with $T=t_{f i n}=2$ at $y=0.875$ and velocity with $v_{1}=-0.6167$ and $v_{2}$ as listed.

\begin{tabular}{lcccccc}
\hline$v_{2}$ & -3.3243 & -1.8892 & -0.6167 & 0.6167 & 1.8892 & 3.3243 \\
\hline$\varepsilon=1 / 4$ & $3.4103 \mathrm{e}-05$ & $5.8409 \mathrm{e}-05$ & $1.5900 \mathrm{e}-04$ & $9.9293 \mathrm{e}-03$ & $2.8628 \mathrm{e}-02$ & $3.6588 \mathrm{e}-02$ \\
$\varepsilon=1 / 8$ & $1.8549 \mathrm{e}-05$ & $3.1806 \mathrm{e}-05$ & $8.7218 \mathrm{e}-05$ & $5.1617 \mathrm{e}-03$ & $1.6729 \mathrm{e}-02$ & $2.0902 \mathrm{e}-02$ \\
$\varepsilon=1 / 16$ & $1.1557 \mathrm{e}-05$ & $1.9875 \mathrm{e}-05$ & $5.5139 \mathrm{e}-05$ & $3.0362 \mathrm{e}-03$ & $1.0753 \mathrm{e}-02$ & $1.2107 \mathrm{e}-02$ \\
$\varepsilon=1 / 32$ & $9.0618 \mathrm{e}-06$ & $1.5652 \mathrm{e}-05$ & $4.4112 \mathrm{e}-05$ & $2.1743 \mathrm{e}-03$ & $8.8528 \mathrm{e}-03$ & $8.8848 \mathrm{e}-03$ \\
\hline
\end{tabular}

in velocity space given by $v_{1}=-0.6167$ and $v_{2}$ as listed in the table. This quantity mimics the behavior of $\iint \hat{f} \psi d x d t$ with $\psi \equiv 1$. Table 3.3 shows the corresponding relative quantity $I(\tilde{f}-f)(y, v, T) / I(f)(y, v, T)$ at the same values of $T, y$, and $v$. The convergence rate is not uniform for all velocities. For those components $f_{k}$ that correspond to velocities pointing towards the wafer surface, i.e., with $v_{2}<0$, the main portion of the information travels from the given inflow condition, and convergence is good. For the other components corresponding to information traveling back up from the wafer surface, i.e., with $v_{2}>0$, both absolute and relative errors deteriorate somewhat.

\section{REFERENCES}

[1] H. Babovsky, Derivation of stochastic reflection laws from specular reflection, Transport Theory Statist. Phys., 16 (1987), pp. 113-126.

[2] A. G. Belyaev, On Singular Perturbations of Boundary Problems, Ph.D. thesis, Moscow State University, Moscow, 1990 (in Russian).

[3] T. S. Cale, T. H. Gandy, and G. B. Raupp, A fundamental feature scale model for low pressure deposition processes, J. Vac. Sci. Technol. A, 9 (1991), pp. 524-529.

[4] C. Cercignani, The Boltzmann Equation and Its Applications, Appl. Math. Sci. 67, SpringerVerlag, New York, 1988.

[5] A. Friedman and B. Hu, A non-stationary multi-scale oscillating free boundary for the Laplace and heat equations, J. Differential Equations, 137 (1997), pp. 119-165.

[6] A. Friedman, B. Hu, And Y. Liu, A boundary value problem for the Poisson equation with multi-scale oscillating boundary, J. Differential Equations, 137 (1997), pp. 54-93.

[7] M. K. Gobbert, T. S. Cale, And C. A. Ringhofer, One approach to combining equipment scale and feature scale models, in Process Control, Diagnostics, and Modeling in Semi- 
conductor Manufacturing, M. Meyyappan, D. J. Economou, and S. W. Butler, eds., The Electrochemical Society Proceedings Series, vol. 95-4, 1995, pp. 553-563.

[8] M. K. Gobbert, T. S. Cale, And C. A. Ringhofer, The combination of equipment scale and feature scale models for chemical vapor deposition via a homogenization technique, VLSI Des., 6 (1998), pp. 399-403.

[9] M. K. Gobbert, T. P. Merchant, L. J. Borucki, and T. S. Cale, A multiscale simulator for low pressure chemical vapor deposition, J. Electrochem. Soc., 144 (1997), pp. 3945-3951.

[10] M. K. Gobbert and C. Ringhofer, Mesoscopic scale modeling for chemical vapor deposition in semiconductor manufacturing, in Dispersive Transport Equations and Multiscale Models, N. B. Abdallah, A. Arnold, P. Degond, I. Gamba, R. Glassey, C. D. Levermore, and C. Ringhofer, eds., IMA Volumes in Mathematics and its Applications 136, Springer-Verlag, New York, 2003, pp. 133-150.

[11] M. K. Gobbert and C. A. Ringhofer, An asymptotic analysis for a model of chemical vapor deposition on a microstructured surface, SIAM J. Appl. Math., 58 (1998), pp. 737-752.

[12] M. K. Gobbert, C. A. Ringhofer, and T. S. Cale, Mesoscopic scale modeling of microloading during low pressure chemical vapor deposition, J. Electrochem. Soc., 143 (1996), pp. 2624-2631.

[13] M. K. Gobbert, S. G. Webster, J.-F. Remacle, and T. S. Cale, A Spectral Galerkin Ansatz for the Deterministic Solution of the Boltzmann Equation on Irregular Domains, Technical report, University of Maryland, Baltimore County, Baltimore, MD, 2002.

[14] H. Grad, On the kinetic theory of rarefied gases, Comm. Pure Appl. Math., 2 (1949), pp. 331407.

[15] A. Kersch and W. J. Morokoff, Transport Simulation in Microelectronics, Progress in Numerical Simulation for Microelectronics, 3, Birkhäuser Verlag, Basel, 1995.

[16] A. KLAR, A numerical method for kinetic semiconductor equations in the drift-diffusion limit, SIAM J. Sci. Comput., 20 (1999), pp. 1696-1712.

[17] C. R. Kleijn and C. Werner, Modeling of Chemical Vapor Deposition of Tungsten Films, Progress in Numerical Simulation for Microelectronics, 2, Birkhäuser Verlag, Basel, 1993.

[18] T. P. Merchant, M. K. Gobbert, T. S. Cale, and L. J. Borucki, Multiple scale integrated modeling of deposition processes, Thin Solid Films, 365 (2000), pp. 368-375.

[19] C. Ringhofer, Computational methods for semiclassical and quantum transport in semiconductor devices, Acta Numer., 6 (1997), pp. 485-521.

[20] C. Ringhofer, Space-time discretization of series expansion methods for the Boltzmann transport equation, SIAM J. Numer. Anal., 38 (2000), pp. 442-465.

[21] C. Ringhofer, C. Schmeiser, And A. Zwirchmayr, Moment methods for the semiconductor Boltzmann equation on bounded position domains, SIAM J. Numer. Anal., 39 (2001), pp. 1078-1095.

[22] C. Schmeiser and A. Zwirchmayr, Convergence of moment methods for linear kinetic equations, SIAM J. Numer. Anal., 36 (1998), pp. 74-88.

[23] S. G. Webster, M. K. Gobbert, J.-F. Remacle, and T. S. Cale, Parallel numerical solution of the Boltzmann equation for atomic layer deposition, in Euro-Par 2002 Parallel Processing, B. Monien and R. Feldmann, eds., Lecture Notes in Comput. Sci. 2400, Springer-Verlag, Berlin, 2002, pp. 452-456. 